Reprod. Nutr. Dévelop., 1980, 20 (1 B), 235-240.

\title{
Ontogenèse des relations hypothalamo-hypophyso-testiculaires chez le fœtus de lapin
}

par G. VEYSSIÈRE, M. BERGER, Christiane JEAN-FAUCHER, M. DE TURCKHEIM, CI. JEAN

Physiologie Comparée ef Endocrinologie, Université de Clermont II,

Les Cézeaux, B. P. 45, 63170 Aubière, France.

Summary. Ontogenesis of hypothalamo-hypophyseal-testicular interrelationships in the fetal rabbit.

Hypothalamo-hypophyseal-testicular inferrelationships were studied in the fetal rabbit during sexual organogenesis (from days 20 to 25 of gestation). Significant amounts of plasma and testicular testosterone appeared at day 19 of gestation. In organ culture, the 20-day old fetal testis was showed to secrete testosterone in the medium. The pituitary of the 20-day old male fetus contained biologically active gonadotropic factor (s) since testicular production of testosterone was stimulated when both testis and pituitary were placed together in organ culture. From 20 to 23 days, embryonic hypophyseal secretion of gonadotropic factors did not seem to be regulated by circulating androgens. It has been hypothesized that the negative feed-back of circulating androgens started acting at 24 or 25 days of gestation.

\section{Introduction.}

II est actuellement bien établi, dans certaines espèces de mammifères, que le testicule fœtal synthétise ef sécrète de la testostérone (revue : Bloch, 1979) et que les gonadostimulines sont présentes dans l'hypophyse et/ou le sang des fœtus (revue : Jenkin, Mc Millen et Thorburn, 1979). Chez l'homme, l'hypothalamus fœetal contient du LHRH (Aubert, Grumbach et Kaplan, 1977). Toutefois, au cours de la différenciation sexuelle, les relations hypothalamo-hypophyso-testiculaires ont été peu étudiées. L'hypophysectomie fœtale, pratiquée avant le début de l'organogenèse sexuelle, induit une déficience testiculaire chez le lapin (Jost, 1948). Chez le fotus de rat, la testostérone (T) pourrait agir par rétroaction sur la sécrétion de LH (Chouraqui, Zeis et Weniger, 1977).

Le présent travail a pour but de répondre à 2 questions : 1) l'hypophyse fœtale est-elle capable de stimuler la synthèse de $T$ par le testicule dès le début de l'organogenèse sexuelle ? 2) à ce stade, les androgènes circulant chez le fœetus contrôlent-ils la sécrétion des gonadostimulines fœtales? 


\section{Matériel et méthodes.}

Nous utilisons des lapins Néo-Zélandais. L'heure de la copulation représente le temps zéro de la gestation.

1. - Détermination quotidienne de la $T$ testiculaire et plasmatique de 18 à 25 jours de gestation.

2. - Recherche d'une activité gonadotrope de l'hypophyse fœtale. Dans un premier temps, nous avons voulu savoir si à 20 jours (début de l'organogenèse sexuelle), l'hypophyse des fœtus mâles était capable de stimuler la production de $T$ par le testicule. Six moitiés de testicules prélevés sur des fœtus de 20 jours, sont cultivées $24 \mathrm{~h}$ seules $(N=6)$ ou en présence de 6 hypophyses $(N=4)$ provenant des mêmes animaux. Les cultures sont effectuées sur $700 \mu$ de milieu synthétique 199 additionné de pénicilline $(250 \mathrm{UI} / \mathrm{ml})$ et de sérum de veau (10 p. 100) d'après la méthode de Morat, Chevalier ef Dufaure (1971).

3. - Modification expérimentale du taux circulant des androgènes chez le fœtus. Immunisation. - Des lapines sont immunisées activement contre la $T$ par injection d'un complexe T-3-O-Carboxymethyl Oxime-albumine bovine, et mises au mâle 2 mois plus tard. Le pourcentage de liaison de la $T$ et de la dihydrotestostérone (DHT) est mesuré, par dialyse à l'équilibre (Forest, Rivarola et Migeon, 1968).

Injection de DHT. 3 injections de DHT (dans de l'huile de sésame) sont administrées à $12 \mathrm{~h}$ d'intervalle à des lapines gestantes sacrifiées $5 \mathrm{~h}$ après la dernière injection à $20,22,23$ et 25 jours. La DHT a été choisie de préférence à la T ou à l'cestradiol en raison des effets directs de ces hormones sur le testicule (Dufau ef al., 1978). La T est mesurée par RIA (Veyssière ef al., 1976).

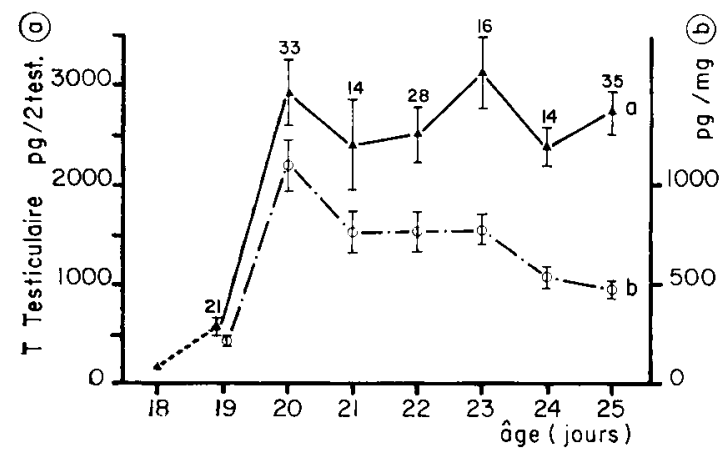

FIG. 1 .

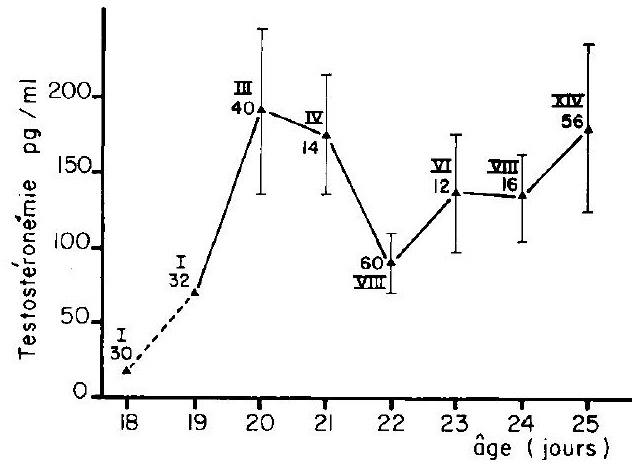

FIG. 2 .

FIG. 1. - Evolution de la testostérone festiculaire ( $a=$ contenu, $b=$ concentration) chez des foefus âgés de 18 d̀ 25 jours. Valeur moyenne avec erreur-type ef nombre de mesures. A 18 jours une seule mesure sur un pool de 28 gonades des deux sexes.

FIG. 2. - Evolution de la festostéronémie chez des faefus âgés de 18 à 25 jours. Valeur moyenne avec erreur-type. A chaque stade nombre de pools mesurés en chiffres romains, nombre d'échantillons par pool en nombres arabes. A 18 jours le pool comprend des plasmas des deux sexes. 


\section{Résultats.}

1. - Evolution de la $T$ testiculaire ef plasmatique (fig. 1 et 2). - A 18 jours, dans un pool de 28 gonades mâles et femelles, la T mesurée est très faible (166 pg). De 18 à 19 et de 19 à 20 jours, la $T$ s'élève rapidement dans le testicule dont le contenu à 20 jours (2930 $\pm 320 \mathrm{pg} / 2$ testicules) ne varie plus jusqu'à 25 jours ( $2715 \pm 225 \mathrm{pg} / 2$ testicules). La concentration testiculaire de $\mathrm{T}$, maximum à 20 jours $(1096 \pm 130 \mathrm{pg} / \mathrm{mg})$ varie peu jusqu'à 23 jours $(783 \pm 84 \mathrm{pg} / \mathrm{mg})$ et décroît de 23 à 25 jours $(485 \pm 41 \mathrm{pg} /$ $\mathrm{mg})$.

Des concentrations significativement mesurables $(68 \mathrm{pg} / \mathrm{ml})$ de $\mathrm{T}$ apparaissent, dans le plasma, à 19 jours. Entre 20 et 25 jours, période de l'organogenèse sexuelle, la testostéronémie ne présente pas d'élévation caractéristique (fig. 2).

2. - Culture organotypique de testicules et d'hypophyses (fig. 3). - Après $24 \mathrm{~h}$ de culture, des testicules prélevés sur des fœtus de 20 jours libèrent dans le milieu des quantités importantes de $\mathrm{T}(110,2 \pm 8,6 \mathrm{ng} / 3$ testicules $)$. Le contenu testiculaire, après $24 \mathrm{~h}$ de culture $(7,5 \mathrm{ng} / 3$ testicules), est le double de celui mesuré in vivo $(3,6 \mathrm{ng} /$ 3 testicules à 21 jours).

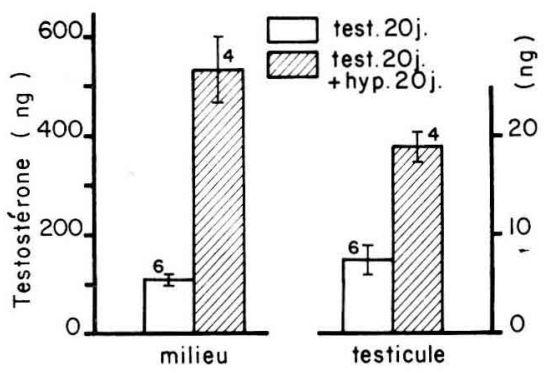

FIG. 3. - Production de testostérone par des testicules de fotus de 20 jours cultivés $24 \mathrm{~h}$ en présence ou en absence d'hypophyses mâles de même âge. 3 testicules sont cultivés seuls ou associès à 6 hypophyses. Valeur moyenne avec erreur-type et nombre de mesures.

Si les testicules sont associés pendant $24 \mathrm{~h}$ à des hypophyses prélevées sur des fotus mâles de même âge ( 20 jours), la T est significativement multipliée par 4,8 dans le milieu et par 2,4 dans les testicules.

3. - Immunisation de la mère. - Chez des mâles prélevés à 21 jours sur des mères immunisées, la $\mathrm{T}(99,4 \pm 0,05$ p. 100) et la DHT $(99,7 \pm 0,1$ p. 100) sont presque entièrement liées aux protéines plasmatiques. Chez des témoins de même âge les pourcentages de liaison sont de 73,6 $\pm 1,2$ p. 100 pour $T$ et de 77,8 $\pm 0,5$ p. 100 pour $\mathrm{DHT}$. Les anticorps anti-T de la mère passent donc chez le fotus et bloquent les androgènes circulants.

Le contenu testiculaire en T est analogue chez les témoins $(2405 \pm 450 \mathrm{pg} / 2$ testicules) et les immunisés ( $2170 \pm 220 \mathrm{pg} / 2$ testicules). Il en est de même des concentrations testiculaires de T: $770 \pm 110 \mathrm{pg} / \mathrm{mg}$ chez les témoins, $890 \pm 110 \mathrm{pg} / \mathrm{mg}$ chez les immunisés.

4. - Injection de DHT à la mère (tableau 1). - L'injection de DHT induit, à tous les stades, une élévation de la DHT dans le plasma fotal. A 25 jours, l'injection de 3 fois Img de DHT réduit significativement la T testiculaire. A 23 jours, la même dose 
induit, chez le fotus, un taux identique de DHT circulante mais ne modifie pas le contenu testiculaire en T. A 20 et 22 jours, une élévation considérable de la DHT circulante, chez le fœtus, est sans effet sur la T testiculaire.

\section{TABLEAU 1}

Effets de l'injection de DHT à la mère sur la $T$ testiculaire du fœtus

\begin{tabular}{|c|c|c|c|c|}
\hline \multirow{2}{*}{$\begin{array}{c}\text { Age } \\
\text { (jours) }\end{array}$} & \multirow{2}{*}{ Traitement } & \multirow{2}{*}{$\begin{array}{c}\text { DTH plasmatique } \\
\text { fœiale } \\
(\mathrm{pg} / \mathrm{ml})\end{array}$} & \multicolumn{2}{|c|}{ T testiculaire } \\
\hline & & & $\mathrm{pg} / 2$ test. & $\mathrm{pg} / \mathrm{mg}$ test. \\
\hline \multirow{2}{*}{20} & $\begin{array}{l}\mathrm{DHT} 3 \times 10 \mathrm{mg} \\
\quad(\mathrm{N}=9)\end{array}$ & $5980 \pm 1410 *$ & $2390 \pm 460$ & $775 \pm 141$ \\
\hline & $\begin{array}{l}\text { Témoins } \\
(N=16)\end{array}$ & $50 \pm 10$ & $3040 \pm 530$ & $1040 \pm 198$ \\
\hline \multirow{2}{*}{22} & $\begin{array}{c}\text { DHT } 3 \times 10 \mathrm{mg} \\
(\mathrm{N}=7)\end{array}$ & $5420 \pm 1345 *$ & $2430 \pm 410$ & $566 \pm 69$ \\
\hline & $\begin{array}{l}\text { Témoins } \\
(\mathrm{N}=34)\end{array}$ & $61 \pm 5$ & $1990 \pm 240$ & $570 \pm 78$ \\
\hline \multirow{2}{*}{23} & $\begin{array}{c}\mathrm{DHT} 3 \times 1 \mathrm{mg} \\
(\mathrm{N}=16)\end{array}$ & $295 \pm 50 *$ & $2560 \pm 350$ & $483 \pm 91 *$ \\
\hline & $\begin{array}{l}\text { Témoins } \\
(\mathrm{N}=16)\end{array}$ & $40 \pm 10$ & $3125 \pm 350$ & $783 \pm 84$ \\
\hline \multirow{2}{*}{25} & $\begin{array}{c}\mathrm{DHT} 3 \times 1 \mathrm{mg} \\
(\mathrm{N}=13)\end{array}$ & $290 \pm 30 *$ & $1120 \pm 200 *$ & $209 \pm 38 *$ \\
\hline & $\begin{array}{l}\text { Témoins } \\
(\mathrm{N}=30)\end{array}$ & $38 \pm 8$ & $2460 \pm 210$ & $460 \pm 45$ \\
\hline
\end{tabular}

$N=$ nombre de fotus. Les valeurs sont les moyennes \pm l'erreur type.

* Significativement différent des témoins de même âge.

\section{Discussion.}

Chez le fotus de lapin, la synthèse et la sécrétion de T débutent entre 18 et 19 jours ; elles précèdent la différenciation de l'appareil génital, notamment celle des gonoductes qui s'effectue après le 20e jour (Jost, 1947).

En culture organotypique, des hypophyses prélevées sur des fœłus mâles de 20 jours augmentent significativement la production de $T$ par des testicules de fœtus de même âge. Ceci montre qu'au $20^{e}$ jour de gestation, l'hypophyse des fœtus mâles contient un ou plusieurs facteurs gonadotropes, biologiquement actifs à la fois sur la synthèse et la libération de T par le testicule. L'élévation de la T testiculaire ef sanguine, à 19 jours, pourrait être consécutive à l'apparition ou à l'intensification de l'activité gonadotrope de l'hypophyse embryonnaire. Des expériences complémentaires, effectuées avec des hypophyses de 18 à 19 jours, permettront d'élucider ce problème. Chez le fotus de lapin, les effets de la décapitation précoce (19e jour) sur l'organo- 
genèse sexuelle (Jost, 1948) et la présence, à ce stade, d'une activité gonadotrope dans l'hypophyse, sont en faveur du rôle de cette glande dans le contrôle de la fonction endocrine du testicule. Des études sont en cours pour déterminer la nature de ce facteur gonadotrope et le rôle exact de l'hypophyse dans le déclenchement ef la régulation de l'activité endocrine du testicule fœetal. De la LH biologiquement active et/ou immunologiquement réactive a été mesurée, pendant et après la période de la différenciation sexuelle, dans l'hypophyse fœetale (Jenkin, Mc Millen et Thorburn, 1979).

Nos résultats montrent également qu'au $20^{\circ}$ jour de la gestation, le testicule est capable de répondre à une stimulation hypophysaire gonadotrope qui pourrait être de la LH car, in vitro, au même stade, HCG stimule la production de T par le testicule qui contient des récepteurs spécifiques de LH-HCG (George ef al., 1978).

Les modifications expérimentales du taux circulant des androgènes chez le fœtus ont des effets variables selon l'âge. Chez des fotus mâles de 21 jours dont la mère a été immunisée, la $T$ testiculaire n'est pas modifiée, ce qui indique qu'à cet âge, une très forte diminution des fractions libres de la $T$ ef de la DHT sanguines est sans effet sur le système hypothalamo-hypophysaire. Chez des nouveau-nés de mères immunisées, une réduction analogue du taux des androgènes non liés élève significativement la $T$ testiculaire (Veyssière et al., 1978). Au $25 \mathrm{e}$ jour de la gestation, l'injection de $\mathrm{DHT}$ réduit significativement la $\mathrm{T}$ testiculaire, probablement en déprimant la sécrétion des gonadostimulines de l'hypophyse embryonnaire. A 20, 22 et 23 jours, un traitement similaire est inefficace. Les cultures organotypiques nous ont montré que, dès le stade de 20 jours, l'hypophyse fotale possède une activité biologique de type LH. La sécrétion de cette hormone ne semble pas contrôlée par les androgènes circulants avant le $23^{\mathrm{e}}$ ou le $24^{\mathrm{e}}$ jour. Si l'on admet que le rétrocontrôle des androgènes sur la sécrétion de LH s'exerce via l'hypothalamus (Sharp et Fraser, 1978), il semble qu'au cours de cette période, la sécrétion de LH soit indépendante de l'hypothalamus. Cette hypothèse est en accord avec le fait que, si l'on sépare l'hypophyse de l'hypothalamus, à cef âge, l'organogenèse sexuelle mâle n'est pas affectée (Jost, 1970). Le contrôle par l'hypothalamus des sécrétions hypophysaires nécessite certaines conditions dont une ou plusieurs ne seraient pas remplies : présence de récepteurs spécifiques des androgènes, existence des relations neurovasculaires hypothalamo-hypophysaires, sécrétion de LHRH. Chez le fœtus de lapin, le plexus primaire du système porte hypophysaire se développe entre 20 ef 23 jours (Campbell, 1966) el les extraits hypothalamiques sont, au 20e jour, dépourvus d'activité LHRH (Campbell et Gallardo, 1966).

Chez le fotus de lapin, il semble donc exister entre 20 et 23 jours (période d'organogenèse sexuelle), une sécrétion gonadotrope autonome de l'hypophyse, non régulée par les androgènes circulants. On peut concevoir que, parallèlement au développement des relations anatomiques hypothalamo-hypophysaires (20-23 jours), il y ait, sous l'effet des androgènes circulants, une maturation progressive du gonadostat hypothalamique, de sorte qu'entre 23 et 25 jours la sécrétion des gonadostimulines hypophysaires pourra être régulée. Ce feed-back négatif des androgènes reste fonctionnel jusqu'à la naissance (Veyssière et al., 1978) et pourrait expliquer la diminution des androgènes testiculaires qui apparaît dès 24 jours et s'accentue en fin de gestation (Veyssière et al., 1976). 


\section{Références}

AUBERT M. L., GRUMBACH M. M., KAPLAN S., 1977. The ontogenesis of human fetal hormones IV. - Somatostatin, luteinizing hormone releasing factor, and tyrotropin releasing factor in hypothalamus and cerebral cortex of human fetuses $10-22$ weeks of age. J. clin. Endrocrinol. Metab., 44, 1130-1141.

BLOCH E., 1979. Fetal gonadal activity and reproductive tract differentiation, 21-37. In ZONDEK T., ZONDEK L. H., Fetal Endocrinology, S. Karger, Basel (Switzerland).

CAMPBELL H. J., 1966. The development of the primary portal plexus in the median eminence of the rabbit. J. Anat., 100, 381-387.

CAMPBELL H. J., GALLARDO E., 1966. Gonadotrophin-releasing activity of the median eminence at different ages. J. Physiol., 186, 689-697.

CHOURAQUI J., ZEIS A., WENIGER J. P., 1977. Corrélations hypophyso-testiculaires chez l'embryon de rat. C. R. Acad. Sci., Paris, Sér. D, 285, 1475-1478.

DUFAU M. L., HSUEH A. J., CIGORRAGA S., BAUKAL A. J., CATT K. J., 1978. Inhibition of Leydig cell function through hormonal regulatory mechanisms, 193-239. In HANSSON V., RITZEN M., PURVIS K., FRENCH F. S., Endocrine opproach to male contraception, Scriptor Copenhagen.

FOREST M. G., RIVAROLA M. A., MIGEON C., 1968. Percentage binding of testosterone, androstenedione and dehydroisoandrosterone in human plasma. Steroids, 12, 323-343.

GEORGE F. W., CATT K. J., NEAVES W., WILSON J. D., 1978. Studies on regulation of testosterone synthesis in fetal rabbit testis. Endocrinology, 102, 665-673.

JENKIN G., MC MILLEN I. C., THORBURN G. D., 1979. The development of fetal hypothalamicpituitary gonadal adrenal function, 58-90. In ZONDEK T., ZONDEK L. H., Fetal Endocrinology S. Karger, Basel (Switzerland).

JOST A., 1947. Recherches sur la différenciation sexuelle de l'embryon de lapin. I. - Embryologie génitale normale. Arch. Anat. micr. Morphol. exper., 36, 151-200.

JOST A., 1948. Influence de la décapitation sur le développement du tractus génital ef des surrénales de l'embryon de lapin. C. R. Soc. Biol., 142, 273-275.

JOST A., 1970. Hormonal factors in the development of the male genital system, 11-18. in ROSEMBERG E., PAULSEN C. A., The human testis. Plenum Press, New York.

MORAT M., CHEVALIER M., DUFAURE J. P., 1971. Culture in vitro de fragments de testicules de porc : étude morphologique et histoenzymologique. C. R. Soc. Biol., 165, 1894-1898.

SHARP P. J., FRASER H. M., 1978. Control of reproduction, 271-332. In JEFFCOATE S. L., HUTCHINSON J. S. M., The endocrine hypothalamus, Acad. Press, New York.

VEYSSIËRE G., BERGER M., JEAN-FAUCHER Ch., DE TURCKHEIM M., JEAN Cl., 1976. Levels of testosterone in the plasma, gonads, and adrenals during fetal development of the rabbit. Endocrinology, 99, 1263-1268.

VEYSSIĖRE G., BERGER M., JEAN-FAUCHER Ch., DE TURCKHEIM M., JEAN Cl., 1978. Demonstration of negative feed-back of circulating androgens in the rabbit fetus at the end of gestation. IRCS Med. Sci., 6, 313. 